In: Hoffmann, Dagmar u.a. (Hrsg.): Mediensozialisationstheorien. Neue Modelle und Ansätze in der Diskussion. Wiesbaden: VS Verlag für Sozialwissenschaften, 2007. S. 67-91

\title{
Mediensozialisation: Entwicklung von Subjektivität in medialen und kulturellen Figurationen
}

Ben Bachmair

\section{Sozialisation in medialen und kulturellen Figurationen}

Sozialisation meint die nachhaltige und typische Persönlichkeitsentwicklung in Gesellschaften. Ob dabei intentionale Sozialisationsformen wie Schulunterricht und Familienerziehung einzuschließen sind, wird erst dann wichtig, wenn sich in spezifischen historischen Situationen solche intentionalen Formen als wesentlich für die Persönlichkeitsentwicklung herausstellen, z.B. als beabsichtigte Konsumoder Medienerziehung in Schule und Familie oder in Alltagsvollzüge eingebundenen Bewältigungsstrategien, Verlockungen der Werbung mit verfügbares Geld in Einklang zu bringen. „Sozialisation“ von „Enkulturation“ abzugrenzen mag hilfreich sein, wenn man sich in der Lage sieht „Gesellschaft" und „Kultur“ begrifflich und von den Phänomenen her gesehen auch angemessen auseinander zu halten, was zumindest für unsere Gesellschaft nicht möglich ist. Hinzukommt, dass der folgenden Erörterung ein Verständnis von Kultur als Gestaltung zugrunde liegt, die vor allem im gestalteten Leben erscheint. Kultur als Gestaltung meint individuelle und kollektive Gestaltungsprozesse, die zu kulturellen Produkten führen, was unterschiedliche Phänomene wie Institutionen und Medien einschließt. Mit dieser auf Gestaltung und ihre Produkte (anderes formuliert: die kulturellen Objektivationen) ausgerichteten Betrachtung von Gesellschaft und Kultur lohnt es sich „Sozialisation“ als Schlüsselkategorie zu nutzen, um das Verhältnis von Medien und dem vorrangigen Persönlichkeitstypus der heutigen Menschen zu entfalten.

Für unsere aktuelle historische Situation mit ihrer Individualisierungsdynamik ist vorrangig, dass die prägenden Institutionen die Menschen als Subjekte denken. „Selbstverwirklichung“ und ,individuelle Verantwortung“ sind dafür die Schlagworte, ,Formen jugendlicher Vergemeinschaftung heute“ (Hitzler/Bucher/ Niederbacher 2001) liefern dazu die Forschungsfragen. In der britischen Diskussion hat Anthony Giddens (1991) die Subjektivität (,Self-Identity“) als den we- 
sentlichen Faktor der aktuellen gesellschaftlichen Entwicklung am Ende der Moderne (,late modern age") untersucht, und damit eine Gesellschaftsanalyse zur Sozialisationstheorie der Entstehung und Bedingung von Subjektivität vorgenommen ${ }^{1}$. Mit dem Gedanken der Persönlichkeitsentwicklung als Subjektivität ist das Konzept eines Persönlichkeitstypus in der Diskussion, das herausarbeiten will, wie Menschen mit ihrer typischen Wahrnehmung, mit ihren typischen Emotionen, mit ihrem typischen Handeln in dieser gesellschaftlich kulturellen Welt leben und sie damit auch herstellen.

Mit diesem Konzept geht es nicht darum einem vereinfachen Modell wie dem der Medien als Sozialisationsinstanz ,mehr' Dimensionen einzubauen. Vorsicht ist deshalb bei einem konzeptionellen Einstieg in das Thema Mediensozialisation geboten, das mit dem Gedanken der Medien als ,wichtiger Sozialisationsinstanz" beginnt, wie das der aktuelle Überblick von Fritz, Sting und Vollbrecht (2003) tut. Für die Erklärung der nachhaltigen und typischen Persönlichkeitsentwicklung unserer Gesellschaft wäre so gesehen der Frage nach „mächtigen“ Sozialisationsinstanzen der Industriegesellschaft, z.B. die der Schule, die Frage nach der Sozialisationsfunktion der heutigen Medien und ihrer Repräsentationsstrategien anzufügen. Mit solch einem Modell, in dessen Mittelpunkt prägende gesellschaftliche Institutionen und ihre Mechanismen stehen, das mit einem passiv gedachten Subjekt versucht zu erklären, wie denn die nachwachsende Generation entwickelt wird, wie sich der bestimmende Teil der Elterngeneration einer Gesellschaft die Kindergeneration wünscht, wäre jedoch wenig Typisches für unsere Gesellschaft untersucht, denn in unserer Gesellschaft haben die einzelnen Menschen als Individuen und Subjekte eine dynamische Funktion. So gesehen ist deshalb nicht vorrangig die Frage nach den Medien als ,wirkmächtige" Instanzen zu stellen und wie sie die Kinder- oder Jugendgeneration nachhal-

1 Giddens geht dabei Fragen nach wie „The Self: Ontological Security and Existential Anxiety, S. $35 \mathrm{ff}$., The Trajectory on the Self”, S. 70ff., „Sequestration of Experience”, S.144ff., „Tribulations of the Self”, S. 181. In der „critical discourse analysis“ hat Gunther Kress auf diese Fragestellungen hingewiesen: Linguistic Processes in Sociocultural Practice. London (OUP) 1989 (first published in Australia by Deakin University Press in 1984), chapter 2 ,Speech and speakers: the formation of individuals in discourse and genre“, pp. 33 - 51. Insbesondere die folgende Definition von Kress S. 37 ist hier von Interesse: „In the short term a reading position is constructed by a discourse, which provides instructions about how to read a text or a set of texts. That instruction is always also an instruction to act in certain ways, to take stances, to conform or to adapt. In the longer term these constantly reiterated demands construct certain 'subject positions', that is, sets of statements which describe and prescribe a range of actions, modes of thinking and being, for an individual, compatible with the demands of a discourse. In that way we learn how to be men and women, husbands or wives, sons or daughters, mothers or fathers, secretaries or bosses, teachers or policemen, lovers or casual friends". 
tig zu einem spezifischen Persönlichkeitstyp entwickeln. Wesentlich wichtiger wäre die Frage, an welchem gesellschaftlichen Ort - das ist für unsere Gesellschaft das Alltagsleben - sich Mediensozialisation ereignet.

In der Perspektive dieses Erkenntnis leitenden Bildes beschäftigt sich deshalb eine Theorie oder Empirie der Mediensozialisation mit dem Verhältnis der Menschen als Subjekt zu ihrer gesellschaftlich konstruierten Wirklichkeit. Die deutsche Diskussion stellt mit dem Bild des „Aufwachsens in Medienwelten“ die gesellschaftliche Wirklichkeit in den Mittelpunkt: Medien bilden dann ein Feld oder Felder der gesellschaftlichen Wirklichkeit, die wiederum Wirklichkeit schafft. In diesem Kontext ist auch nicht mehr das Generationenverhältnis von tragender Wichtigkeit für Sozialisation, da zum vorrangigen Persönlichkeitstyp der Wandel, das ist die Fähigkeit der Akkommodation und Adaptation an und in wechselnden gesellschaftlichen Feldern, gehört.

\section{Medien als gewichtiger Teil einer kulturellen Figuration}

Medien und Sozialisation sind also auf spezifische Weise in unsere Kultur verwoben, z.B. in die Kinderkultur mit typischen Familienformen, Konsumgewohnheiten, Lebensräumen, Formen von Massenkommunikation. Raymond Williams hat dafür den Begriff der „,cultural form“ (Williams 1975) ${ }^{2}$ geprägt. Für die 1970er Jahre sah er dabei die über Fernsehen vermittelte Technologisierung im Vordergrund, deren Dynamik und Erklärungsmuster er aufzudecken versuchte. Ein gleichfalls bestimmendes Moment der kulturellen Figuration war die Verknüpfung eines dominanten Mediums, nämlich des Fernsehens, mit dem Alltagsleben (vgl. Silverstone 1994, Charlton/Bachmair (Hrsg.) 1990, Hepp 1998) mit Fragen nach Konsum und der damit einhergehenden Veränderung der Beziehung von Öffentlich und Privat ${ }^{3}$. In der augenblicklichen Diskussion unserer kulturellen Situation stehen die Umbrüche der Industriegesellschaft im Vordergrund mit den Gedanken der Individualisierung, Fragmentierung, Alltagsästhetisierung und damit einhergehenden neuen Erlebnisweisen. Aktuelle Mediensozialisation vollzieht sich dann in der kulturellen Figuration der entwickelten Industriegesellschaft und einer ihrer dominanten Formen, nämlich der der Massenkommunikation. Im Mittelpunkt steht die Persönlichkeitsentwicklung von

2 In diesem Text wird der Begriff der „cultural form“ in Anlehnung an das Konzept von Elias (1970) mit „kulturelle Figuration“ übersetzt.

3 Siehe Silverstone 1994: 52: „The suburbanisation of the public sphere”. Die Grundlagen dieses Themas hat Jürgen Habermas (1962) erarbeitet. 
Kindern und Jugendlichen innerhalb der spezifischen Kulturform einer medial vermittelten Massenkommunikation.

\subsection{Massenkommunikation als Prozess diskursiver Praktiken im Schema des Encoding und Decoding: Stuart Hall}

Stuart Hall (1980, deutsch 1999) bestimmt Massenkommunikation, und das ist einer der Mediensozialisation bestimmenden Prozesse, als „miteinander verbundene Praktiken“ (1999: 93), deren Gegenstand, wie er formuliert, „Bedeutungen und Nachrichten in Gestalt besonderer Zeichenträger, die wie jede Kommunikations- oder Sprachform mittels Kodeoperationen im Rahmen der syntagmatischen Kette eines Diskurses organisiert sind" (ebd.). Ein Medien-Produkt zirkuliert bzw. wird in verschiedenen Öffentlichkeiten verteilt. Dies ist eine „,diskursive Form“, also ein „Diskurs, der dann funktioniert, wenn er in ,gesellschaftliche Praktiken umgewandelt wird" (ebd.). Medien mit ihrem Programm sind Teile eines „sinntragenden Diskurses“ (1999: 97), die die Teilnehmer an der Massenkommunikation in Bedeutungszusammenhängen der Medien- und Programmproduktion herstellen (encoding) und in Bedeutungszusammenhängen der Mediennutzung, und das ist vor allem der Alltag, rezipieren, d.h. aneignen (decoding). Wenn beispielsweise Kinder den Fernseher einschalten, dann klinken sie sich in den Encoding-Diskurs ein und eröffnen den Decoding-Diskurs. Das ist möglich, weil ein Programmangebot zum Einschalten, zum Kaufen oder für andere Nutzungsformen zur Verfügung steht.

\subsection{Medien als Objekte des Alltagslebens und die Aneignung objektivierter kultureller Muster}

Mediennutzung ist Teil des Alltagslebens und seiner inneren Logik. Das bestätigen für Deutschland die regelmäßigen „Basisuntersuchungen zum Medienumgang“" von Kindern und Jugendlichen, die zeigen (Medienpädagogische Forschungsverbund Südwest 2003: 5 f.), dass Fernsehen, die schulischen Anforderungen (Hausaufgaben und Lernen), Freunde treffen, draußen und drinnen spielen, Musikkassetten und CDs hören, Aktivitäten mit Familie und Eltern, Sport usw. eine Einheit eingegangen sind.

Dieser Gedanke der Medien als Alltagsobjekte folgt dem Sozialisations- und Aneignungsmodell der Kulturtheorie von Norbert Elias (1937). Alltagsobjekten wie Messer oder Gabel sind Eigenschaften eingeschrieben, die sich deren Benutzer bei der Benutzung aneignen können. Auch moderne elektronische Medien 
sind kulturelle Objekte, in die kulturelle Erfahrungen einschrieben ${ }^{4}$ sind, die sich die Nutzer aneignen können. Der Einfluss der medialen Angebote auf die Persönlichkeitsentwicklung von Kindern und Jugendlichen ist also möglich, weil sich Kinder die medialen Inhalte, deren Repräsentationsweisen und deren Nutzungssituation und was damit in diesen kulturell objektiviert ist (z.B. körperliche Passivität vor dem TV-Bildschirm) aneignen. Das sieht z.B. zurzeit so aus, dass nur Bücher und komplexe Spielsoftware aufwändige und ergänzende schulische Lernprozesse notwendig machen. Ohne diese institutionelle Vermittlung, also schon mit dem Akt des Einschaltens oder mit dem Kauf eines Mediengerätes oder einer Software klinken sich die Nutzer in den jeweiligen Typus von Massenkommunikation und ihre sozialen Praktiken, anders formuliert, in deren bedeutungsgeleitende Diskurse ${ }^{5}$ ein - so das Modell von Stuart Hall - innerhalb derer sie sich auf die kulturell notwendigen Aneignungsprozesse einlassen. Sozialisation ,ereignet' sich also in der Handlungsbeziehung eines Menschen mit einem Medium oder einem Medien-Set innerhalb einer spezifischen kulturellen Figuration. So gehen Kinder beispielsweise mit kulturell neuen Genres wie Pokèmon um, indem sie mit anderen Kindern deren Merchandising-Produkte tauschen, dies jedoch in der Bundesrepublik im Rahmen einer Art von Konsumund Medien-Kindheit (um ein Schlagwort der Einfachheit halber zu verwenden) tun, die jedoch auch Kinder- und Familienarmut einschließt, die dann in der Schule zu erheblichen Spannungen führt.

\section{Vom Prägemodell zum Modell der Dynamik subjektiver Aneignung}

Grundlage des Konzeptes von Sozialisation ist die Beziehung eines Menschen zu seiner Umwelt, in der sich die Persönlichkeit entwickelt. Diese Umwelt konstituiert sich immer sozial, kulturell und dinglich. Selbstverständlich gehört zur sozialen, kulturellen und dinglichen Umwelt notwendigerweise eine Generationenbeziehung, wofür Familie und Schule typisch sind. Aber auch die Medien werden nahezu ausschließlich von der Erwachsenengeneration produziert. Mit Blick auf Mediensozialisation geht es sicherlich erst einmal um Medien als Teil der Umwelt eines Menschen. Damit ist noch wenig über die Beziehung von Kindern

4 Theoretisch angemessener ist, statt von „eingeschrieben“ zu sprechen, den Gedanken der kulturellen symbolischen Objektivationen systematisch zu entfalten. Vgl. dazu Bachmair 1996: 40ff., $58 \mathrm{ff}$.

5 Dieses Konzept der vorrangigen Herausbildung von Subjektivität und Bedeutung hat George H. Mead (1934) in seiner Untersuchung zur Beziehung von „Geist, Identität und Gesellschaft“ (mind, self and society) noch an der medien-freien Interaktionsbeziehungen herausgearbeitet hat. Werner Loch (1974) fasst den Beitrag des Symbolischen Interaktionismus für die Untersuchung von Sozialisation zusammen. 
bzw. Jugendlichen und Medien ausgesagt, z.B. über die Geschichten, die Medien erzählen und die sich ein Kind individuell angeeignet hat, indem es möglicherweise aus Hauptfiguren Nebenfiguren, aus dem Verlierer den Gewinner macht, Gewaltdarstellungen übertreibt oder für sich nicht zulässt. Es bleibt auch noch unbestimmt, was Persönlichkeit und Subjektivität konkret meint. In einer Militärgesellschaft wäre schon das Wort Persönlichkeit unangemessen, geschweige denn das Phänomen einer ,eigenverantwortlichen Persönlichkeit“, die der heutige Jugendmedienschutz als Ziel formuliert. Zudem gilt es, die aktuelle Medienentwicklungen systematisch einzuordnen: Was heißt „Medien als Teil der Umwelt eines Menschen", wenn die Menschen bei Casting-Shows vom Typ Pop Stars oder Big Brother nicht mehr der fertigen Sendung gegenüber sitzen, sondern zu deren Protagonisten werden, zumindest prinzipiell, um danach wieder ins zuschauende Publikum zurückzukehren?

In der kulturhistorischen und gesellschaftlichen Entwicklung sind ebenso wie in der theoretischen Modellierung unterschiedlichste Ausprägungsformen von „Persönlichem“, von „Entwicklung“, „Umwelt“ und Beziehung zwischen Menschen und Umwelt möglich. Für die Beziehung zwischen Menschen und ihrer Umwelt, also auch für ihre mediale Umwelt, sind seit Platon und Aristoteles zwei Paradigmen denkbar, eine "mimetisch“ abbildende und eine ,poetisch“ gestaltende Beziehung. Ein Blick zurück in die neuere Theoriegeschichte zeigt, wie sehr noch Anfang der 70er Jahre des letzten Jahrhunderts, also zu einer Zeit in der Persönlichkeitsentwicklung und Entfaltung von Individualität in der Bundesrepublik für wichtig genommen wurden, das Modell von Prägung, Übernahme und Reproduktion im Vordergrund des Sozialisationskonzeptes stand ${ }^{6}$. So stellte der Schulpädagoge Helmut Fend (1974) als "theoretische Orientierung“ die folgende „Doppelfunktion des Sozialisationsprozesses“ heraus: „Reproduktion der Gesellschaft und Aufbau der Persönlichkeit" (S. 11). Es ging also darum, „wie im gesellschaftlichen Prozess der Reproduktion das Individuum als soziales Wesen entsteht und welche Rückwirkung dieser Prozess auf die Bewahrung oder Veränderung de Gesellschaft und ihrer Subgruppen hat" (Fend 1974: 13). Überträgt man diese Argumentation, in dessen Mittelpunkt die Dynamik der Reproduktion, also die mimetischen Prägung steht, auf die Mensch-MedienBeziehung, dann fallen theoriegeschichtliche Parallelen zur frühen Medienwir-

6 Von der Kritik ,prägetheoretischer Komponenten" geht Hurrelmann (1998: 13) aus. Er zeigt, dass der französische Soziologe Emile Durkheim vor etwa einem Jahrhundert das Konzept der Sozialisation im Sinne eines Prägemodells einführte (S. 13). Durkheims Ansatz ging davon aus, dass die bei der Geburt „asozialen“ menschlichen Wesen zum „sozialen Leben“ geführt würden. Er stellt die leitenden Sozialisationsmodelle vor (S. 20ff.): Das „mechanistische“, das „organismische“, das „systemische“ und das „interaktive Model“. 
kungsforschung auf, bei der ein aktiver Sender passive Rezipienten beliefert und analog zu den gesendeten Informationen und Botschaften beeinflusst (Lasswell 1948). Jedoch noch vor der theoretischen Wende in der Sozialisationstheorie hin zu einem Modell der gestaltenden Persönlichkeit in ihrer Lebenswelt als Grundlage von Sozialisation wandte sich die innovative Medienwissenschaft dem Modell des aktiven Mediennutzers zu, der nicht ,abgefüllt' wird. Im so genannten Uses-and-Gratifications-Approach begannen Jay G. Blumler und Elihu Katz (1974) die Mediennutzer als Subjekte des massenmedial vermittelten Kommunikationsprozesses herauszustellen. Menschen sehen fern, weil sie damit etwas bekommen oder erreichen wollen. Wissenschaft sah in der Aktivität des Fernsehens eine Form sozialen Handels, das sich auch auf die mediale Umwelt richtet und diese mediale Umwelt zum Teil der subjektiven Lebenswelt macht. Diese subjektive Lebenswelt ist immer eine soziale Welt und deswegen angefüllt mit Zeichen und Bedeutungen, wozu in der augenblicklichen kulturellen Figuration der entwickelten Industriegesellschaft industriell produzierte und als Konsumobjekt individuell verfügbare Medien gehören.

\section{Alltagsobjektivationen, Subjektivität und Lebenswelt : Norbert Elias und Alfred Schütz}

Die von Norbert Elias (1937) Mitte der 1930er Jahre im englischen Exil erarbeitete Form der Persönlichkeits- und Subjektivitätsentwicklung zeigt, wie oben schon kurz skizziert, wie in Alltagsobjekte, z.B. Messer oder Gabel, kulturelle Erfahrungen, z.B. Aggressionskontrolle oder der Distanz, eingeschrieben sind, die sich die Nutzer von Messer und Gabel dann eignen können. Norbert Elias skizzierte diese Dynamik an der Veränderung des Persönlichkeitstyps zu Beginn der Renaissance, die mit einem sich verändernden Alltagsleben und neuen Alltagsobjekten, z.B. beim Essen, einherging. Er fragte, wie denn die moderne Persönlichkeit des sich selbst organisierenden und selbst kontrollierenden Subjekts entstand. Diese war bzw. ist als Persönlichkeitstyp Voraussetzung für eine funktionierende Industriegesellschaft, in der sich Arbeiter und Angestellte selber bei ihrer zielorientierten Arbeit kontrollierend anleiten. Wichtig war dabei die Distanz, die sich im Alltagsleben u.a. mit der Gabel prägend mit der lebenswichtigen Ernährung ausprobieren und einüben lässt. In der Wahrnehmung lief diese Distanzierung über die Zentralperspektive. Zentralperspektivisch gemalte Bilder stellen ihre Betrachter der Welt gegenüber, wobei der Bildaufbau eine räumlich organisierte Welt im Kopf des Betrachters entstehen lässt bzw. der Betrachter lernt, den Bildaufbau in eine eigene räumliche Vorstellung umzusetzen. Bildbetrachter waren von da ab nicht mehr Teil der Welt, sondern konnten als distanziert Wahrnehmende Konstrukteuren einer Welt sein. Dabei begangen die Men- 
schen, sich auch in ihren eigenen Wahrnehmungsmittelpunkt zu stellen. Mit dem Alltagsgegenstand Messer übten sie zudem die Selbstkontrolle insbesondere die über ihrer aggressiven Wünsche und Strebungen. Parallel dazu übernahm der Staat das Gewaltmonopol. Im Sinne dieser Entwicklung von Persönlichkeit und Subjektivität in der kulturellen Dynamik sind Medien wie Fernsehen Alltagsobjektivationen, die in die Beziehung der Menschen zu sich selber sowie zu sozialen und dinglichen Umwelt eingreifen. Diese Dynamik von Alltagsobjektivationen und Subjektivität bildet die systematische Grundlage, um Massenkommunikation und ihre Medien in ihrer Sozialisationsfunktion einzuschätzen. Subjektivität konstituiert sich dabei überindividuell in einer kulturellen Dynamik, die ihrerseits vom vorfindlichen Typus der Subjektivität geprägt wird. In dieser Dynamik spielt Massenkommunikation mit industriell erstellten und verbreiteten Textangeboten sowie individueller Rezeption und Nutzung im Alltag eine gewichtige Rolle.

\section{Aneignung und Entäußerung als Basisdynamik der Lebenswelt}

Das Konzept der phänomenologischen Soziologie (Alfred Schütz' Arbeiten und die Zusammenfassung bzw. Weiterführung von Berger und Luckmann 1970) hebt die Trennung zwischen dem Materialen und dem zeichenhaft Symbolischen von Kommunikation auf, weil es all das, was die Menschen schaffen und sich aneignen, in Beziehung zum Schaffen und Aneignen setzt. Wirklichkeit ist damit etwas Fassbares und Gegenständliches in einem Prozess. Die Menschen stellen Wirklichkeit in sozialen Situationen und auf der Basis der vorfindlichen gegenständlichen und sozialen Welt her und eignen sie sich auch wieder an. Das Modell spannt Wirklichkeit zwischen die beiden Pole 'Handeln' und 'institutionelle Wirklichkeit'. Wirklichkeit unterscheidet sich im Grad der Verdichtung und Verbindlichkeit. Medien sind als symbolische Objektivationen Teile und Bausteine der Wirklichkeit, je nachdem, ob man sich Ergebnis oder Prozess von Herstellung und Aneignung anschaut.

„Das menschliche Ausdrucksvermögen besitzt die Kraft der Objektivation, das heißt, es manifestiert sich in Erzeugnissen menschlicher Tätigkeit, welche sowohl dem Erzeuger als auch anderen Menschen als Elemente ihrer gemeinsamen Welt 'begreiflich' sind. Objektivationen durch Ausdruck sind mehr oder weniger dauerhafte Indikatoren subjektiver Empfindungen. Sie ermöglichen deren ,Begreifbarkeit' über die Vis-à-vis-Situation, in welcher sie unmittelbar erfaßt werden können, hinaus. Zum Beispiel kommt das subjektive Gefühl von Zorn in der Vis-à-vis-Situation direkt in mancherlei physischen Merkmalen zum Ausdruck: Mienenspiel, Ausfallstellung des Körpers, bestimmte Bewegungen der Arme und Füße und so weiter. Diese An- 
zeichen für Zorn sind faßbar, ,während' die Vis-à-vis-Situation andauert. Deshalb bietet sie die optimale Möglichkeit, zum Anderen als Subjekt Zugang zu erlangen. Solche Ausdrucksbewegungen sind allerdings nicht fähig, die leibhaftige Gegenwärtigkeit der Vis-à-vis-Situation zu ,überdauern' Zorn kann jedoch mittels einer Waffe vergegenständlicht, beziehungsweise objektiviert werden." (Berger/Luckmann 1970: 36 ff.).

\begin{abstract}
Modell der Sozialisation als produktive Verarbeitung der inneren und der äußeren Realität

Im Blickwinkel der Sozialisationstheorie geht es an dieser Stelle der theoretischen Argumentation darum, diese Form der sozialen und sinnbezogenen Mediennutzung in die soziale Welt hineinzustellen, die immer eine Welt der Elterngeneration und der Kindergeneration ist. Diese theoretische Aufgabe hat im deutschen Sprachraum Klaus Hurrelmann in den 1980er Jahren zu einem Modell verdichtet, in dessen Mittelpunkt Persönlichkeitsentwicklung als Entwicklung von Subjektivität gedacht ist. Diesem theoretischen Modell liegt selbstverständlich auch eine andere Dynamik in Gesellschaft und Kultur zugrunde. Also nicht nur die Theorie sondern auch das Phänomen Sozialisation hat sich geändert.
\end{abstract}

Klaus Hurrelmann (1986) fasst den aktuellen und vorherrschenden wissenschaftlichen Diskurs zusammen, indem er sich auf die Menschen bezieht, die sich als Subjekte interaktiv und sinnhaft in einer Sozialwelt entwickeln; ja, nicht nur entwickeln, sondern diese Sozialwelt mitgestalten und mit verändern. Menschen beziehen sich sowohl auf ihre soziale, kulturelle und dingliche Umwelt als auch auf ihre psychisch körperliche Innenwelt der Gefühle, Träume, Körperempfindungen, Erfahrungen und Lebensziele. Medien gehen in diese Beziehungen eines Menschen zu sich selber und seiner Innenwelt, sowie zu seiner Umwelt ein. Diese Beziehung kann für Mitglieder einfacher oder komplexer sozialen Gruppen, von Peer-Gruppen bis zu Schichten, Szenen oder altersspezifischen Generationen typisch oder auch individuell einmalig sein. In Rezeptionsstudien bzw. in Studien zur Mediennutzung in sozialen Kontexten wie dem Alltag zeigt sich der Vermittlungszusammenhang von Medien, subjektiver Innenwelt und sozialer, kultureller, dinglicher Außenwelt. 
Basismodell der Sozialisation:

Medien gehen ein in die Beziehung der Kinder bzw. Jugendlichen

- $\quad$ zu sich selber: subjektive Innenwelt

- $\quad$ zu anderen: soziale Umwelt

- zur Welt der Dinge und Ereignisse

- zur Welt der Kultur

Medien können in dieses Verhältnis eingehen z.B. als das dingliche Gerät, wie die Fernbedienung, die ein Junge seinem Vater wegnehmen möchte, um selber über die Programmwahl der Familie zu bestimmen. Den eigenen Fernseher zu besitzen, das Passwort für den Internetzugang zu haben, kann der Indikator und die dingliche Botschaft an die Umgebung sein, nicht mehr als klein und schutzbedürftig gelten zu wollen. Zum Funktionszusammenhang „Medien“ gehören weiterhin die Programmangebote, z.B. die Geschichte auf dem Bildschirm, das Spiel auf dem Gameboy. Abstrakt formuliert sind dies die Repräsentionen und Repräsentationsformen, z.B. der Bildschirm, die Puppe zu den Teletubbies, das Life-Ereignis und die Internet-Seite zu Big Brother. Zu den medialen Funktionszusammenhängen gehören die Nutzungssituation (z.B. allein vor dem Bildschirm / im Chat mit einer anonymen Gruppe) und die dingliche Seite der Medien, also das Instrument Gameboy. Dies sind alles mediale Funktionselemente außerhalb eines Subjektes.

\section{Bedeutungskonstitution, Aneignungs- und Handlungsmuster}

Dem Sozialisationsprozess liegt eine Aneignungsdynamik zugrunde, die zu beschreiben sich vom Alltag und der psychosozialen Entwicklung der Kinder bzw. Jugendlichen her theoretisch beschreiben lässt. Ralf Vollbrecht (2003: 15) unterscheidet zwischen

- situativen Funktionen von Medien: Information, Unterhaltung, Vertreiben von Langeweile (Zeitfüller), Stimmungsregulierung, Eskapismus (Flucht aus dem Alltag), Habitualisierungsfunktion (Zeitstrukturierung)

- $\quad$ sozialen Funktionen der Medien: Gesprächsanlässe in Familien und Peer groups, Meinungsbildung, Gruppenidentität (z.B. in Jugendkulturen), sich in Medienwelten positionieren

- $\quad$ und biographischen, Ich-bezogenen Funktionen von Medien: Identitätsentwicklung (Vorbilder, Normen, virtuelle Erprobung); Selbst-Vergewisserung, Selbst-Reflexivität und Selbst-Darstellung; Modell-Lösung für persönliche oder entwicklungsbezogene Themen. 
Der Überblick von Judith van Evra (2004: $3 \mathrm{ff}$.) stellt nicht die Medienfunktionen, sondern die des sozialen Lernens (Social Learning, Social Cognitive Theory), der Kultivierung (Cultivation Theory) und des persönlichen Nutzen des Mediengebrauches (Uses and Gratifications Theory). Beide Systematisierungsversuche stellen in einem Diskursmodell von Massenkommunikation analytisch den Zusammenhang von Kindern bzw. Jugendlichen und Medien im Kontext des Alltagshandeln her. Dieser Handlungskontakt von Menschen und Medien in Situationen des Alltags, die in die Diskurse der Massenkommunikation eingebunden sind, bilden die dynamische Grundlage von Mediensozialisation. Diese medialen Funktionselemente sind ebenfalls Bestandteile der subjektiven Innenwelt, z.B. in den Fernsehbildern, die sich ein Kind angeeignet hat und von denen es seinen Freunden erzählt. $\mathrm{Zu}$ diesen angeeigneten medialen Funktionselementen gehören auch nicht verbalisierbare Wünsche, eine Hardware zu besitzen oder die Befürchtung, nicht genug Wertschätzung in der Familie zu haben, um so viel vom Familienbudget abzubekommen, um einen Gameboy oder einen eigenen CD-Brenner zu haben. Kinder und Jugendliche gehen mit diesen mediale Funktionselementen als Teil ihrer bedeutsamen, anders formuliert, als Teil ihrer persönlich relevanten sinnhaften Innen- und Außenwelt um. Dies ist nur möglich, indem zu und mit den Bildern auf dem Bildschirm bis hin zur Mitgliedschaft bei Fan-Gruppe, zu der jemand gehört, Bedeutung kommuniziert wird? ${ }^{7}$.

Modell der Bedeutungskonstitution als Handlungskern von Mediensozialisation:

Bedeutung entsteht in Perspektiven, Bezugsfeldern und in Aneignungs- und Entäußerungsprozessen:

- $\quad$ Themen der Menschen in ihrem Lebenslauf (subjektive Sinnperspektive),

- $\quad$ Bilder, Figuren, Geschichten von Medien (Bezugsfeld der Angebote, Repräsentationen und Repräsentationsformen),

- Medien-Erlebnisse (was subjektiv angeeignet ist),

- $\quad$ Situationen der Nutzung von Medien und der Rezeption von Medienangeboten (Aneignungssituationen und Aneignungsformen),

- $\quad$ Bezugsfeld: Soziale Umgebung (Familie und Gleichaltrige),

- $\quad$ Bezugsfeld: intertextuelle Welt der Medien.

7 Dies sind theoretische Annahmen, die auf dem so genannten Symbolischen Interaktionismus George H. Mead (1934, siehe auch Blumer 1973) und dem Sinn bezogenen und Sinn schaffenden Handeln in der subjektiven Lebenswelt (Alfred Schütz 1934, 1984) beruhen. Den medienwissenschaftlichen Bezugsrahmen bietet dafür das Modell der Massenkommunikation als Encoding und Decoding (Stuart Hall 1980), eine theoretische Erklärung von Medien als spezielle Formen von Texten und eine sozialkulturelle Zeichentheorie der Mediensemiotik (Kress, van Leeuwen 1996, 2001, Stuart Hall 1997). 
In dieser Bedeutung konstituierenden Beziehung zwischen Medien und Kinder bzw. Jugendlichen entwickelt sich das Verhältnis dieser Kinder bzw. Jugendlichen zu sich und ihrer sozialen, dinglichen und kulturellen Umwelt. Sie strukturiert Subjektivität als individuelle Form dieser Beziehung. Dieses Verhältnis von Innenwelt und Außenwelt stellen Kinder und Jugendlichen wie alle Menschen her, indem sie etwas von sich nach außen mitteilen (Entäußerung) und indem sie sich etwas kommunikativ aneignen. Wie sieht Medienaneignung ${ }^{8}$ aus, wie verändert sie sich?

\section{Bezugsfelder: Soziale Umgebung (Gleichaltrige) und intertextuelle Welt der Medien}

Beispielsweise hat Heinz Hengst (1985) sich in den 1980er Jahren angesehen, über welche Aneignungsformen beispielsweise ein Film wie E.T. mit dem $B M X$ Fahrrad zusammenhängt. In der Schlussepisode von E.T. entziehen sich die Film-Kinder der verfolgenden Polizei, indem sie mit geländegängigen Fahrrädern von der Straße wegfahren, die Erwachsenen dagegen mit ihren Autos auf der Straße feststecken bleiben. In der Folge des Filmerfolges von E.T. gab es eine riesige Nachfrage nach $B M X$-Rädern, der zu einer eigenen Sportszene führt, wobei das $B M X$-Fahrrad und dessen Fans auch schon vorher, sozusagen als Startmöglichkeit, vorhanden waren. Heinz Hengst erklärt dies mit „latenten Bedürfnissen“ die verbunden mit „medialen Impulsen“ zu einem „Script“ führt, das zu „Freizeitbetätigung“, „Körpererfahrung und Selbstdarstellung“ führt.

\section{Entwicklungslinie: Themen der Menschen in ihrem Lebenslauf, die subjektive Sinnperspektive}

In einem Gespräch von zwei Jungen in einer Grundschule zeigt sich eine andere Form der Filmaneignung, die von der aktuellen Sinnperspektive, den handlungsleitenden Themen zweier Jungen ausgeht. Einer der beiden Jungen, Sven, fühlt sich sehr bedroht fühlt, er greift die vielfältigen Bedrohungsthemen von E.T. auf und teilt mit diesen Filmthemen seine Befindlichkeit seinem Mitschüler mit. Bei einer genauen Analyse zeigt sich, wie dieser Junge sich selektiv auswählend das für ihn Relevante aus dem Film gezogen hat und die entsprechende Filmsymbolik als sprachliches Mitteilungsinstrument einsetzt. Er bezieht eine Lösung zu seinem Gefühl bedroht zu sein, aus dem Film E.T., indem er auf die Anbahnung einer Freundschaft mittels Smarties verweist (Bachmair 1993). 
Bezugsfeld: Bilder, Figuren, Geschichten von Medien und Medien-Erlebnisse

Bei der Einführung der Teletubbies, ein Vorschulprogramm für die unter 3Jährigen hat Maja Götz (1999) die Aneignungsmuster erhoben. Weil dies Programm auch schon für Zweijährige konzipiert war, dies den gängigen Vorstellungen vom angemessenen Alter für die so genannten Fernsehanfänger abweicht, war die Frage nach der Aneignung relevant, um sicher zu stellen, dass diese Altersgruppe nicht überfordert ist. Überforderung ist eine Kategorie, die recht deutlich auf die Generationenbeziehung bei der Mediensozialisation verweist, an die, wie auch beim kodifizierten Jugendmedienschutz, kulturell geprägte Vorstellungen von den für die Persönlichkeitsentwicklung angemessene bzw. unangemessene Mediennutzung geknüpft ist. Maya Götz bat Eltern, ihren Kindern mit einer Videokamera zuzuschauen, wenn die Teletubbies liefen. Zusammenfassend zeigten sich folgende Handlungsweisen in Bezug auf die Sendung:

- Kinder kommentieren z.B. die Sonne und erklären anwesenden Erwachsenen oder Kinder, was sie sehen.

- $\quad$ Sie singen mit oder sprechen mit, was die Tubbies sagen.

- Sie verfolgen gebannt, was in der Sendung zu sehen ist.

- Sie antworten auf eine Aufforderung z.B. mit „Hallo“ auf das BegrüßungsHallo der Kinder eines Realfilms.

- Sie wissen nach wenigen Sendungen, was im Ablauf der Geschichte als nächstes kommen wird und weisen vorweg darauf hin.

- $\quad$ Sie tanzen mit oder bewegen sich wie die Tubbies.

- $\quad$ Sie fragen die Eltern, weil sie etwas nicht verstanden haben.

- Sie klinken sich aus dem Fernsehen aus und spielen selbständig mit ihren Tubbie-Puppen.

Die einzelnen Teile jeder Sendung ermöglichen typischerweise folgende Aktivitäten von Kindern:

- Während des Vorspanns, bei der Auswahl des Tubbies, auf dessen Bildschirmbauch der Realfilm zu sehen sein wird, oder beim Abschied: $\mathrm{Zu}$ schauende Kinder sprechen, singen oder tanzen mit. Sie sagen vorher, was kommen wird oder antworten.

- Während des Realfilms: Kinder verfolgen, teilweise gebannt, die Vorgänge auf dem Bildschirm. Sie antworten, machen Bemerkungen, erkennen Vorgänge.

- Während der kleinen und großen Geschichten im Teletubbie-Land: Kinder verfolgen teilweise gebannt, was abläuft. Sie sagen etwas voraus, kommentieren, erklären, sprechen mit. 
- Während die Tubbies tanzen und bei der Animation: Kinder tanzen und bewegen sich mit, spielen etwas anderes, fragen nach und kommentieren.

Aneignungsform: Experte eines Medien- und Warenangebotes In diesem Handlungs- und Aneignungsmuster wählen und gewichten Mediennutzer die Fülle von Angeboten, indem sie sich innerhalb ihrer Bezugsgruppe integrierend oder abgrenzend zum Experten machen. Der Gegenstand dieses Musters, die mehr oder weniger leicht verfügbare Medien- und Warenangebote, machen das Experten-Muster für die entwickelte Industriegesellschaft bedeutsam, weil ihm die Wählbarkeit des sozialen Handelns zu eigen ist. Im Alltagsleben lässt sich etwas auswählen, z.B. ein traditionelles Mediengenre wie Star Wars oder einen Event wie Deutschland sucht den Superstar. D.h. die in die Entscheidung der Menschen in ihrem jeweiligen Alltagsleben gegebenen und verfügbaren Medien- und Warenangebote lassen die Kommunikation mit sich und seinen Themen und zugleich mit gewählten anderen, das sind die Peers, zu.

Beispiel: Der neunjährige Marcel ist Experte für $\mathrm{Yu}-\mathrm{Gi}-\mathrm{Oh}$, was sich unter anderem an der präzisen Verwendung von Fachtermini für die Protagonisten sehen lässt:

„Yu-Gi-Oh ist eine Serie, die auf RTL II kommt. Die Helden heißen Yugi, Joey, Tea, Tristan, Seto und Mokuba Kaiba, Bakura und Pegasus. Doch mein Favorit ist Joey wegen seiner Drachenarme, die zwar nicht die Stärksten sind. In schwierigen Situationen helfen ihm seine Freunde Yogi, Tea und Tristan wie im Kampf gegen Rex Rabtor, der Dinosammler. Doch Seto Kaiba ist auch nicht schlecht, da er immer und immer versucht, seinen Bruder Mokuba zu retten.“

\section{Erlebnisweisen im Kontext aktueller kultureller Figurationen des Konsums und individueller Lebenswelten}

Medienbezogene Aneignungs- und Handlungsmuster entsprechen der jeweiligen kulturhistorischen Situation. Darauf verwies Mitte der 1930er Jahre schon Walther Benjamin (1981/1955), als er die Beziehung von Film und Erlebnisweisen von Industriearbeitern diskutierte. Er sah eine positive Entsprechung zwischen den Erfahrungen von Industriearbeitern mit Maschinen und der technisch maschinellen Reproduktion des Films als ein in der Logik der Industrieproduktion funktionierendes Kunstwerk. Technologisch standardisierte Verfahren, die so- 
wohl die Arbeit als auch Freizeit prägen, vermitteln Erlebnisweisen, die für das Verhältnis der Menschen zu sich selber und nach außen bestimmend werden.

Zurück zum $B M X$-Fahrrad, auf das auch der Film E.T. als Zeichen-Reservoir zurückgreift. Heinz Hengst stellt nicht nur die sich gegenseitig verstärkende Entsprechung von Freizeitsport $B M X$-Fahren in Peer-Gruppen mit einem Kino-, Video- und Fernsehfilm fest, sondern ein generelles Muster, das Sozialisation bestimmt. Beim $B M X$-Rad nennt er es „Aktivitätenverbund in eigener Regie (Hengst 1985: 22). In Übereinstimmung mit der Jugendsoziologie formuliert er eine generelle Entwicklung, die mit einer zunehmenden Individualisierung einhergeht, die "Sozialisation in eigener Regie“, die bis zur Selbstdefinition der eigenen Biographie ohne prägende Traditionsvorgaben im Sinne von Bastelbiographien verläuft. (Ferchhoff 1994). Dabei spielen die im oben skizzierten Modell der Bedeutungskonstitution aufgeführten Bezugsrahmen eine wesentliche Rolle, insbesondere die Verbindung von selbst gewählten Sozialformen wie FanGruppen und Lifestyle-Szenen, die mit Medien unterschiedlicher Repräsentationsformen im Sinne von Anlässen und Kristallisationskernen gestaltbar werden ${ }^{9}$. In diesen sozial-medialen Räumen, die sich in einem Zwischenfeld von Privatheit und Öffentlichkeit erstrecken, entwickeln sich Erlebnisweisen, beispielsweise im Fall von Cliquen zu Horrorvideos, sozialintegrativ mit körperbezogenen Ängsten umzugehen.

Bei der augenblicklichen medienkulturellen Veränderung entstehen mit Medien und Konsumangeboten komplexe, jedoch integrierte Konglomerate (Medienund Ereignisarrangements), die sich Kinder in neuen Formen der Rezeption aneignen (Aneignungsmuster) und $\mathrm{zu}$ denen sie spezifische Handlungsmuster entwickeln. Mit der Ausdifferenzierung vertrauter Medien wie Fernsehen und deren Verschachtelung mit einer Fülle nicht-medialer Angebote bekommen Kinder textartig verbundene Arrangements, die sie in Prozessen der Bedeutungskonstitution subjektiv sinnvoll aufnehmen und verändern. Da in der Dynamik kultureller Veränderungen der Funktionswandel der Medien mit der Entstehung neuer Erlebnis- und Handlungsweisen einhergeht, mag die Verdichtung dieser Dynamik zu einem Modell hilfreich sein, um nach der sich herausbildenden Subjektivität der Kinder- und Jugendgeneration zu fragen. Ausgangspunkt ist die Einbindung von Medien in Handlungsmustern, Gefühlslagen und subjektive Lebenswelten.

9 Anregendes Material bieten die Untersuchungen von Waldemar Vogelgesang $(1991,2003) \mathrm{zu}$ Video-Cliquen und LAN-Partys. 
(A) Es gibt einen Funktionswandel bei den Medien:

- Vom kohärenten Einzelmedium zu Medien- und Ereignisarrangements.

Programmerfolge wie Pokémon verdeutlichen eine wesentliche Veränderung im Medienbereich. Sie zeigen, dass das in sich geschlossene, kohärente Einzelmedium wie der erzählende Hollywood-Film unwesentlich wird. Es entstehen zunehmend mehr Arrangements aus verschiedenen Medien, Kaufprodukte und Events. Im Arrangement Pokémon hatte die tägliche Fernsehserie vor allem die Funktion, ein Gemeingut für Kinder zu schaffen, einen Bezugsrahmen für die wichtigen Elemente (das Spiel mit dem Gameboy, die Tauschkarten, Magazine, Plakate usw.), der zeigt, was wichtig und was unwichtig ist. So gesehen ist auch klar, dass zwar zeitweise fast dreiviertel aller fernsehenden Kinder die Sendung sahen, dennoch das Spiel auf dem Gameboy für sie maßgeblich blieb. Sie suchten in der Fernsehserie auch nicht die Cartoon-Geschichte. Mit dem Erfolg von Pokémon kamen dann Film und Video ergänzend hinzu.

- Medien werden Provider symbolischer Materialien für die Alltagswelt.

Sicherlich besteht noch die Vorstellung, Kindermedien sollten eigentlich Geschichten erzählen und eventuell auch Spiele möglich machen. Vorrangig ist für heutige Kindermedien dagegen vor allem Material für Handlungsmuster, Gefühlsmuster u.ä. anzubieten. Dazu kann auch Spielen oder Geschichten Erzählen gehören. Mit Materialien wie Fernsehserie, Film, Magazin, Tauschkarten, Gameboy, Websites können Kinder in und mit Mustern leben und in ihrer Welt zurecht kommen, ja, sich eine kleine Welt zurechtlegen. Was das heißt, kann man bei Jugendlichen und ihren Szenen oder Fan-Gruppen sehen. Für Techno-Fans steht bzw. stand beispielsweise die Funktion im Vordergrund, mitten im Arrangement zu sein, sich also im Zentrum von Berlin bei raves mit Hundertausenden mit der richtigen Ausstattung und den richtigen Bewegungen zu inszenieren.

Im Medien- und Ereignisarrangement bekommen Medien vorrangig die Funktion eines „Providers“ symbolischer Materialien, die in alltägliche Lebensvollzüge eingebettet sind. Das führt dann zu so merkwürdigen Konstellationen wie Benetons Plakaten, die vor einiger Zeit wesentliche Lebensthemen wie Sterben, Krankheit und Geburt auf die Litfasssäulen brachten. Die Camel-Werbung ist auch Kaufanreiz, jedoch ebenso Lieferant subtiler Witze für Nichtraucher.

- $\quad$ Im Zirkus der Zeichen - die Verbindung von Zeichen, Bezeichnendem und Bezeichnetem geschieht in verschiedenen Kontexten (Diskursen). 
Phänomene wie Big Brother, Pokémon oder die Plakate von Beneton zu Leben und Tod haben keinen angestammten Bezugsrahmen mehr, innerhalb dessen klar ist, worum es geht, z.B. bei Big Brother um Menschenwürde im Sozialexperiment oder um das exhibitionistische Sozialleben junger Leute von nebenan. Diese Uneindeutigkeit nötigt die Zuschauer, eine bequeme passiv rezeptive Rolle aufzugeben und sich in einen aufwändigen Prozess der ständigen Deutung dessen einzulassen, was da zu sehen und zu hören war (Mikos et al. 2000). Zuschauer, insbesondere Kinder, müssen mit jeder neuen Medienwelle für sich und in ihrer Bezugsgruppe herausbekommen, worum es geht, zum Beispiel ob das Angebot ,in“ oder „out" ist, ob und was sich mit einem Angebot wie Pokèmon anfangen lässt. Erschwerend kommt oft hinzu, dass bei den Medien- und Ereignisarrangements nicht offensichtlich ist, was wichtig und was unwichtig ist. Zudem ist unklar, wie das, was bei den verschiedenen Medien-Bausteinen unmittelbar sichtbar, also was die Medienbausteine auf ihrer Oberfläche anbieten, mit dem zusammenhängt, was eher verborgen angeboten wird. Bei Big Brother führte gerade diese Differenz zwischen dem Offensichtlichen und dem durch Kennerschaft erst zu Erschließenden zu heftigen Debatten. Auch bei den Teletubbies waren sich Eltern und die Öffentlichkeit nicht sicher, was sich für Merkwürdigkeiten bei den Teletubbies denn unter der „niedlichen“ Oberfläche verstecken. Bei Pokémon hat sich die Mehrheit der Schulkinder schnell auf eine Art des Umgehens mit den Angeboten verständigt. Sie integrierten Pokémon in ihre vertrauten Muster des Tauschens, Lernens und Spielens. Den Lehrern bleiben diese Muster jedoch weitgehend verborgen.

(B) Es gibt geänderte Erlebnisweisen bei Kindern:

- $\quad$ Von der aufmerksamen Rezeption zu vielfältigen Aneignungs- und Handlungsmustern.

Komplexe Medien- und Ereigniskonglomerate mit ihrer vielfältigen Mischung medialer Funktionen treffen nicht mehr auf Nutzer, die sich einen Text werkgetreu und im Sinne des Autors oder Regisseurs aneignen. Mit der Ausdifferenzierung der Medien ändern sich Rezeptions- bzw. Nutzungsformen. So entwickelte sich Fernsehen vom Zuschauen im Familienkreis zum Zappen. Sich durch die Fülle des Angebots durchzuzappen ist die Vorlage für komplexe Handlungsmuster, in die Mediennutzung eingebettet ist. Es entstehen vielfältige Muster der Rezeption, der Verwendung und der weiteren Aneignung von Medien und sonstiger Konsumangebote. Mediennutzung ist dabei eingebunden in vielfältige Muster des Alltagslebens und der Lebensführung. Bei oder mit Pokémon können Kinder tauschen, alleine 
spielen, im Internet recherchieren, sich zum Experten entwickeln, $150 \mathrm{Na}$ men von Protagonisten auswendig lernen, Spielzüge wie „Attacken“ lernen, ihr Kinderzimmer ausstatten, Geld für die Spielkonsole, für den Gameboy herbeischaffen, aber auch sich nur vor der Fernsehsendung ausruhen.

- Decoding: Bedeutungskonstitution als ständig zu leistende Nutzeraktivität. Medien strukturieren sicherlich immer noch Mediennutzung, jedoch vorwiegend in den Handlungsmustern der Menschen, die sie nutzen. Das wird umso prägnanter, als Medien nur Teile komplexer Arrangements sind. Dann bleibt den Rezipienten gar nichts anderes übrig, als die vielfältigen, geplanten und ungeplanten Angebote miteinander zu verknüpfen. Es ist nicht mehr wie früher mit zwei oder drei Fernsehprogrammen, als die Produktion dem Publikum etwas vorgab, das es rezipieren konnte oder auch nicht. Jetzt schiebt sich eine typische Aktivität der Rezipienten in den Vordergrund, nämlich die vielfältigen Medientexte zusammenzufügen und sozusagen lesbar zu machen. Mediennutzer geben den symbolischen Angeboten Bedeutung, indem sie sie in ihre Handlungsmuster und in ihre Sozialwelt einbinden. Bedeutungskonstitution macht aus einem Konglomerat von Medien, Situationen und Konsumgütern einen persönlich bedeutsamen Text, der für den jeweiligen Nutzer und in seiner Welt lesbar ist.

Die Welt in meinem Sinne: Erlebnisorientiert die Welt erklären und verstehen.

Die für die fortgeschrittene Industrie- und Konsumgesellschaft wichtige und typische Individualisierung verlangt von den Menschen sich ohne traditionelle und allgemeingültige Vorgaben ihre Lebenswelt selber zurecht zu basteln. Die Fan-Gruppe ist dafür eine recht deutliche Form. Dabei bekommen die eigenen, individuellen und subjektiven Maßstäbe ein enormes Gewicht: So wie ich die Welt sehe und erlebe, ist sie nicht nur richtig, sondern nur dadurch wird meine Welt wirklich. Erlebnisorientierung (Schulze 1992) heißt, unsere Gesellschaft und unsere Welt subjektiv wahrzunehmen und auch als persönliche Welt aufzubauen. Und weil dies der vorrangige Modus der Aneignung ist, wird subjektive und Bedeutung konstituierende Aneignung zum objektiven, das heißt allgemeinen Modus, gesellschaftliche Wirklichkeit herzustellen. Sieht man Individuelles und Soziales als die beiden Brennpunkte einer Ellipse, in deren Spannung immer Gesellschaft und konkret einmaliges Lebens eingespannt sind, dann definiert im Moment der Brennpunkt des Individuellen, was das Allgemeine ausmacht. Die Welt wird dadurch jedoch alles andere als asozial, denn Medien sind sowohl Le- 
bensweltbausteine als auch Teile sozialer Inszenierungen wie Fan-Gruppen, Szenen oder Milieus.

Bei einem Medien- und Ereignisarrangement wie $\mathrm{Yu}$-Gi-Oh erscheint diese Erlebnisorientierung in Aneignungsformen wie des Sammelns und Ordnens von Spielkarten oder wie der, Experte in der Pokèmon-Welt der Peers zu werden, indem das lexikalische Wissensangebot der 150 Protagonisten sowie deren typischen Handlungsmuster (Attacken, Entwicklung) für die eigenen Zwecke genutzt werden.

\section{Alltagsästhetik und kulturelle sowie individuelle Bedeutung der Repräsentationsformen}

\section{Alltagsästhetik}

Welche Figurationen sind in der Welt der dominanten Massenkommunikation vorrangig? Was macht die symbolische Welt des Alltags mit Medien und Medienkommunikation aus? Sind es hierarchisch aufgebaute Sinnwelten oder bringt die auf Konsum aufbauende entwickelte Industriegesellschaft eine, auf den ersten Blick spielerisch erscheinende neue Form der Vergesellschaftung, bei der nicht die Produktion, sondern die individuell verfügende Aneignung standardisierter, symbolisch verfasster Objekte im Vordergrund steht? Was darf man sich darunter vorstellen? Sicher die Fan-Gruppe zu etablierten Genres wie Star Wars oder Raumschiff Enterprise, die sich zu kultartigen Inszenierungen treffen, die wie Kongressen von Experten aussehen oder Karnevalveranstaltungen mit Kostümzwang sind oder eine Mischung davon.

Im Mittelpunkt steht ein spezifischer Typ von Erlebnisweise, den Gerhard Schulze (1992) als „Erlebnisrationalität" beschrieben hat. Die Menschen eignen sich die Welt als ihre persönliche Welt in ihrer subjektiven Sicht an. Schlagwort zur Verdeutlichung dazu ist: „Die Welt in meinem Sinne!“ Vorrangiges Strukturelement ist dabei die subjektive Sinnperspektive der Menschen, die ihre Alltagswelt in einer Konsumwelt individuell verantwortlich aufbauen. Die allgemeine kulturelle Entwicklungs- bzw. Sinnperspektive, die George H. Mead in einer langen Tradition stehend mit „Geist" bezeichnet, ist die Konsumwelt, in der Gleichartigkeit und Individualität eine spezielle und Leben wie Kultur prägende Einheit eingegangen sind. Dafür ist fortschreitende Individualisierung und Fragmentierung bei zunehmender Gleichartigkeit des symbolischen Materials der Massenkommunikation kennzeichnend. Eher bildhaft formuliert, lässt sich diese Beziehung als symbolische Welt, als individuelle Wirklichkeit, benennen. 
Es entstehen also heute als symbolische Welten, in denen Medien und Mediennutzungsmuster, Situationen, Handlungs- und Erlebnisweisen eine Einheit eingehen, die von dem Nutzer ausgeht, bei der sich Lebensstilen und Medien durchdringen. Die Gestaltungsfunktion liegt dafür bei den Menschen, die als Rezipienten und Konsumenten in einem Prozess der Bedeutungskonstitution in ihrer individuellen, thematischen Sinnperspektive, bezogen auf ihre konkrete soziale Umgebung, das intertextuell gemischte Medienangebot in die Alltagswelt kommunikativ integrieren. Die intertextuellen und standardisierten Medienangebote werden dabei zunehmend prägender und dominanter, sodass medienspezifische Erlebnis- und Handlungsweisen entstehen. Symbolische Welten sind aktuelle Figurationen, deren subjektive Seite, funktional beschrieben, Prozesse der Bedeutungskonstitution und der Medienkommunikation sind: Die Menschen bauen sich mit den symbolischen Objektivationen der Massenkommunikation wie mit anderen lebensweltlichen Bausteinen (das sind kulturelle Objektivationen) auch ihre Alltagswelt immer in ihrer thematischen Sinnperspektive. Ihre Erlebnis- und Handlungsweisen entsprechen den Bau- und Funktionsmustern, d.h. der Dramaturgie, der Medien und ihrer inneren Verbindung, also der Massenkommunikation. Mit den medien-adäquaten Erlebnis- und Handlungsweisen konstituiert sich auch Subjektivität neu.

Die objektive Seite der symbolischen Welten, auch wieder funktional beschrieben, ist in heftiger Entwicklung begriffen. Prägend ist hierfür die „Ästhetisierung des Alltagslebens", wie sie Schulze (1992) empirisch beschrieben hat. Ästhetisierung mit den vielfältigen symbolischen Konsummaterialien der Industriegesellschaft ist Ausfluss der Individualisierung der modernen Gesellschaft, sozusagen die Basis von Wahlfreiheit und Wahlnotwendigkeit im Konsum. Es werden soziale Figurationen dominant, welche die Subjekt- und die kulturelle ObjektSeite unserer Gesellschaft mittels Medien und industriell hergestelltem symbolischen Material verbinden. Über die Medien überlagert sich die aktuelle Ästhetisierungswelle mit der Medienkommunikation und der Bedeutungskonstitution der Menschen, was die kulturelle Entwicklung in Richtung Fragmentierung und Individualisierung forciert.

\section{Medien in alltagsästhetischen Schemata}

Die Menschen konstruieren sich also ihren Alltag, organisieren ihr Sozialleben und grenzen sich voneinander ab bzw. ordnen sich einander zu mittels den ihnen verfügbaren symbolischen Materialien, die von Medien, Konsumobjekten und aus Situationen stammen. Dabei entstehen soziale Milieus, die Alltagsleben 
strukturieren. Die so genannten Sinus-Milieus ${ }^{10}$ sind auch relevant für das Fernsehpublikums, deren Sender- und Genre-Vorlieben deutlich mit den alltagsästhetischen Milieus zusammenhängen (Nowak/Flaig 2000).

Auch die Fernsehnutzung der Kinder korreliert mit den alltagsästhetischen Milieus der jeweiligen Familien. Katharina Kuchenbuch (2003) hat hierzu festgestellt, dass Kinder aus Milieus mit der Orientierung auf Modernität weniger Fernsehen (ebd.: 4), Kinder aus modernen Unterschicht- und Mittelschichtmilieus am wenigsten öffentlich-rechtliche Fernsehsender schauen, Kinder aus dem sog. Milieu der Hedonisten überdurchschnittlich viel Zeit fernsehen und dabei RTL II und SuperRTL bevorzugen (ebd.: 5f.), und nur ,mit einem Marktanteil von 3,5 Prozent in diesem Milieu sein schwächstes Ergebnis“ (ebd.: 6) erreicht, die Sendung mit der Maus dagegen Favorit der Kinder aus dem intellektuellen Milieu“ ist (ebd.: 7).

In der Logik der alltagsästhetischen Verflechtung von Medien- und Ereignisarrangements entstehen auch spezifische Beziehungen von Kindern und Medien, die mit der Frage nach Typen und Mustern z.B. in den Studien des Fernsehsenders SuperRTL (2000 und 2002) erhoben wurden. Der Blick auf die medienbezogenen Handlungsmuster oder Gefühlslagen zeigt, wie sich Fernsehen in die subjektive Alltagsbewältigung einmischt und damit auch Muster von Subjektivität mit hervorbringt. Dazu aus dieser Studie Handlungsmuster und Gefühlslagen, die mit Medien eine Einheit eingehen:

Typologie der Kinder nach Freizeitmustern (SuperRTL 2000: 58ff.)

- ,die Passiven“ mit „wenig Eigenaktivität“, jedoch mit viel „actionreichem Fernsehkonsum",

- „die Spielkinder, mit viel Spielzeug und Märchengeschichten“,

- $\quad$ „die Intellektuellen“, die sich auf „mehr Wissen für den leistungsorientierten Vorsprung" konzentrieren, „die Gamer mit viel Spiel, Spaß und Spannung“,

- „die Unauffälligen“ mit Tierliebe und Offenheit für Neues,

- $\quad$ „Fun/Action-Kids“. Sie sind ,jung, dynamisch und selten allein“,

- ,die Allrounder“ mit „Führungsanspruch“, „Ecken und Kanten“"

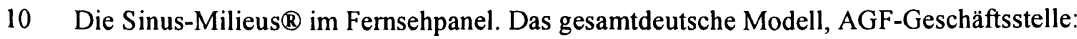
Eschersheimer Landstraße 25-27, 60322 Frankfurt/M., http://www.agf.de. Sinus Sociovision: Ezanvillestraße 59, 69118 Heidelberg, http://www.sociovision.com. GfK Fernsehforschung: Nordwestring 101, 90319 Nürnberg, http://www.gfk.de) 
Diese Muster entstehen, weil für Kinder Medien Elemente in den folgenden Mustern von Gefühlslagen sind: Langeweile / Entspannung / Stimmungsregler bei Wut / Stimmung Traurigkeit / Stimmung Sich Versenken / Geborgenheit vermitteln (SuperRTL 2000: 48 ff.). Ebenso sind Medien in die folgenden Erlebnisdimensionen von Kindern eingebunden, die auch zu einer Differenzierung zwischen verschiedenen Kindertypen führen: Familiäre Geborgenheit und $\mathrm{Zu}$ wendung / Selbstbestimmter Rückzug / Freunde / Positive Spannung und Überraschungen / Lern- und Wissbegierde / Neugier auf andere / Rückzug, Desinteresse und Langeweile / Dramatische Spannungssuche (SuperRTL 2002: 61ff.).

\section{Kulturelle und individuelle Bedeutung von Repräsentationsformen}

Mit der zunehmenden kulturellen Relevanz alltagsästhetischer Muster und Schemata löst sich das Thema oder die „Botschaft“ von einem dafür typischen Medium. So gibt es Pokémon als Fernsehfilm, Spiele-Software, Fan-Magazin, Internet-Site, Tauschkarten oder Poster. Um ein Thema herum entstehen Medien- und Ereignisarrangements, die aus unterschiedlichen medialen Repräsentationsformen bestehen. So hatten die Fernsehbeiträge innerhalb des Medien- und Ereignisarrangement Pokémon die Aufgabe, einen gemeinsamen Bezugsrahmen für die Breitenwirkung von Pokémon herzustellen und damit Bedeutsamkeit zu markieren. Wer als unkundiger Erwachsener versucht, die Fernsehbeiträge als Cartoon zu lesen, musste scheitern, denn da gab es keine traditionelle Geschichte. Auch Geld ist ein Repräsentationsmodus. So zeigt der Geldgewinn bei Wer wird Millionär den Wert dieser Sendung und des dort verhandelten Wissen an, wohingegen die 100.000 DM bei der ersten Big-Brother-Staffel deutlich machten, dass das verhandelte soziale Phänomen doch eher so billig ist wie der Container, in dem es ab lief. Die große Fernsehshow bei Pop Stars hatte im Medienund Ereignisarrangement die Funktion Größe und Bedeutsamkeit herauszustreichen, obwohl sie nur der Werbetrailer für das Hauptprodukt CD-Rom war.

Einzelne Repräsentationsformen wie das Buch, der erzählende Kinofilm oder Fernseh-Trailer haben in unserer Kultur eine eher prägnante und vertraute $\mathrm{Be}$ deutung, andere wie die Telefonnummerneinblendung während einer Fernsehsendung eher nicht. Im jeweiligen Medien-Mix, d.h. im Repräsentations-Mix bleibt also viel Deutungsraum für die Bestimmung dessen, was wohl mit Website, Werbe-Trailer, Telefonrufmöglichkeit, Casting-Show, Zeitungsbericht usw. gemeint ist oder gemein sein könnte. In die Art des Mediums bzw. des Arrangements ist also Bedeutung eingeschrieben, die in individuellen Prozessen der Bedeutungskonstitution re-interpretiert werden. Damit bekommt die Mediennut- 
zung bis hin zum physischen Umgang eine ganz und gar nicht unwesentliche Sozialisationsfunktion.

\section{Literatur}

Bachmair, Ben (1993): Tiefenstrukturen entdecken - Medienanalyse und Massenkommunikation. In: W. Holly/U. Püschel (Hrsg.), Medienrezeption als Aneignung. Methoden und Perspektiven qualitativer Medienforschung. Opladen, S. 43-57.

Bachmair, Ben (1996): Fernsehkultur. Subjektivität in einer Welt bewegter Bilder. Opladen.

Benjamin, Walter (1981): Das Kunstwerk im Zeitalter der technischen Reproduzierbarkeit. 12. Aufl., Frankfurt/M., 1. deutsche Fassung in: Walter Benjamin: Schriften. Frankfurt 1955; Originalausgabe als französische Übersetzung in: Zeitschrift für Sozialforschung, Jg. 5, 1936.

Berger, Peter/Luckmann, Thomas (1970): Die gesellschaftliche Konstruktion der Wirklichkeit. Eine Theorie der Wissenssoziologie. Frankfurt/M.

Blumer, Herbert (1973): Der methodologische Standort des Symbolischen Interaktionismus. In: Arbeitsgruppe Bielefelder Soziologen (Hrsg.), Alltagswissen, Interaktion und gesellschaftliche Wirklichkeit. Bd 1. Symbolischer Interaktionismus und Ethnomethodologie. Reinbek bei Hamburg, S.80-146.

Blumler, Jay G./Katz, Elihu (Eds) (1974): The Uses of Mass Communications. Current Perspectives on Gratifications Research. London.

Charlton, Michael/Bachmair, Ben (1990) (Hrsg.): Medienkommunikation im Alltag - Interpretative Studien zum Medienhandeln von Kindern und Jugendlichen. Schriftenreihe des Internationalen Zentralinstituts für das Jugend- und Bildungsfernsehen, Bd. 24, München.

Claus, Daniel (1981): Theorien der Subjektivität. Einführung in die Soziologie des Individuums. Frankfurt/M.

Durkheim, Emile (1907/1972): Erziehung und Soziologie. Düsseldorf.

Elias, Norbert (1937/1979): Über den Prozeß der Zivilisation. Soziogenetische und psychogenetische Untersuchungen. 6. Aufl., Frankfurt/M.

Elias, Norbert (1970/1990): Was ist Soziologie. 6. Aufl., Weinheim/München.

Evra, Judith van (2004): Television and Child Development. $3^{\text {rd }}$ ed., Mahaw/New Jersey/London.

Fend, Helmut (1974): Gesellschaftliche Bedingungen schulischer Sozialisation. Soziologie der Schule 1. Weinheim/Basel.

Ferchhoff, Wilfried (1994): Individualisierte Kindheit heute. Kindliche Entwicklungsbedingungen im Spannungsfeld von Konsumverhalten, Medienrezeption und öffentlichem Raum am Ende des 20. Jahrhunderts. In: Archiv für Wissenschaft und Praxis der sozialen Arbeit, S. 108-123.

Fritz, Karsten/Sting, Stephan/Vollbrecht, Ralf (Hrsg.) (2003): Mediensozialisation. Pädagogische Perspektiven des Aufwachsens in Medienwelten. Opladen.

Gebauer, Gunter/Wulf, Christoph: Mimesis (1992): Kultur - Kunst - Gesellschaft. Reinbek bei Hamburg.

Giddens, Anthony (1991): Modernity and Self-Identity. Self and Society in the Late Modern Age. Cambridge/Oxford.

Götz, Maya (1999): Begeisterung bei den Kindern, Besorgnis bei den Eltern. In: TELEVIZION 12/2, S. 54-63.

Habermas, Jürgen (1962): Strukturwandel der Öffentlichkeit. Untersuchungen zu einer Kategorie der bürgerlichen Gesellschaft. Frankfurt/M.

Hall, Stuart (Ed.) (1997): Representation. Cultural Representations and Signifying Practices. London.

Hall, Stuart (1980): Encoding/Decoding. In: S. Hall/D. Hobson/A. Lowe/ P. Willis (Eds), Culture, Media, Language. London, pp. 128-139. 
Hengst, Heinz (1985): Selbstbehauptung in der Medienkultur. Zur Eigenständigkeit von Subkulturen - Beispiel: BMX-Fahren. In: medien praktisch, 9. Jg., H. 4, S. 19-22.

Hepp, Andreas (1998): Fernsehaneignung und Alltagsgespräche. Fernsehnutzung aus der Perspektive der Cultural Studies. Opladen/Wiesbaden.

Hitzler, Ronald/Bucher, Thomas/Niederbacher, Arne (2001): Leben in Szenen. Formen jugendlicher Vergemeinschaftungen heute. Opladen.

Holly, Werner/Püschel, Ulrich (Hrsg.) (1993): Medienrezeption als Aneignung. Methoden und Perspektiven qualitativer Medienforschung. Opladen.

Hurrelmann, Klaus (1986/1998): Einführung in die Sozialisationstheorie. Über den Zusammenhang von Sozialstruktur und Persönlichkeit. 6. Aufl., Weinheim.

Kress, Gunter/Van Leeuwen, Theo (1996): Reading Images. The Grammar of the Visual Design. London.

Kress, Gunther/Van Leeuwen, Theo (2001): Multimodal Discourses. The Modes and Media of Contemporary Communication. London.

Kuchenbuch, Katharina (2003): Die Fernsehnutzung von Kindern aus verschiedenen Herkunftsmilieus. In: Media Perspektiven, o. Jg., H. 1, S. 2-11.

Lasswell, Harold D. (1948): The Structure and the Function of Communication in Society. In: L. Bryson (Ed.), The Communication of Ideas. New York, pp. 37-51.

Loch, Werner (1974): Symbolische Bedingungen von Sozialisation. In: Wege zum Menschen. Monatsschrift für Arzt und Seelsorger, Erzieher, Psychologen und soziale Berufe. Göttingen, S. 333-343.

Mead, George, M. (1934): Mind, Self and Society. From the Standpoint of a Social Behaviorist. Chicago.

Medienpädagogischer Forschungsverbund Südwest (2003): Basisstudie zum Medienumgang 6- bis 13-Jähriger. KIM-Studie 2003: Kinder und Medien, Computer und Internet. Baden-Baden.

Mikos, Lothar/Feise, Patricia/Herzog, Katja/Prommer, Elizabeth/Veihl, Verena (2000): Im Auge der Kamera. Das Fernsehereignis Big Brother. Berlin.

Negt, Oskar/Kluge, Alexander (1972): Öffentlichkeit und Erfahrung. Zur Organisationsanalyse von bürgerlicher und proletarischer Öffentlichkeit. Frankfurt/M..

Nowak, Dorothea/Flaig, Berthold (2000): Die Sinus-Milieus im Fernsehpanel. Heidelberg.

Sandbothe, Mike/Marotzki, Winfried (Hrsg.) (2000): Subjekt und Öffentlichkeit. Kulturwissenschaftliche Grundlagenprobleme virtueller Welten. Köln.

Schulze, Gerhard (1992): Die Erlebnisgesellschaft. Kultursoziologie der Gegenwart. Frankfurt/M.

Schütz, Alfred (1932/1974): Der sinnhafte Aufbau der sozialen Welt. Eine Einleitung in die verstehende Soziologie. Frankfurt/M.

Schütz, Alfred/Luckmann, Thomas (1984/1994): Strukturen der Lebenswelt. Bd. 2. Frankfurt/M.

Silverstone, Roger (1994): Television and Everyday Life. London.

SuperRTL Medienforschung: Kinderwelten (2000). Studienbericht Köln (RTL Disney Fernsehen $\mathrm{GmbH} / \mathrm{Co}$. KG). Durchführung der Studie: IJF Institut für Jugendforschung, München. Datenanalyse und Redaktion des Studienberichtes: Transferzentrum Publizistik und Kommunikation.

SuperRTL Medienforschung: Kinderwelten (2002). Freizeit und Medien im alltäglichen Erleben von Kindern. Studienbericht Köln (RTL Disney Fernsehen GmbH/Co. KG). Durchführung der Studie: IJF Institut für Jugendforschung, München. Datenanalyse und Redaktion des Studienberichtes: Transferzentrum Publizistik und Kommunikation.

Vetter, Hans-Rolf (Hrsg.) (1991): Muster moderner Lebensführung. Ansätze und Perspektiven. München.

Vogelgesang, Waldemar (1991): Jugendliche Video-Cliquen. Action- und Horrorvideos als Kristallisationspunkte einer neuen Fankultur. Opladen.

Vogelgesang, Waldemar (2003): LAN-Partys: Jugendkulturelle Erlebnisräume zwischen Off- und Online. In: merz. 47. Jg., H. 5, S. 65-75. 
Vollbrecht, Ralf (2003): Aufwachsen in Medienwelten. In: F. Karsten/S. Sting/R. Vollbrecht (Hrsg.): Mediensozialisation. Pädagogische Perspektiven des Aufwachsens in Medienwelten. Opladen, S. 13-24.

Williams, Raymond (1975/1990): Television: Technology and Cultural Form.. $2^{\text {nd }}$ ed., London.

Winter, Carsten/Thomas, Tanja/Hepp, Andreas (2003) (Hrsg.): Medienidentitäten. Identität im Kontext von Globalisierung und Medienkultur. Köln. 